\title{
Scientific ethics committees: a user's guide
}

\author{
D A Fitzgerald, D Isaacs, A Kemp
}

Arch Dis Child 2005;90:1249-1250. doi: 10.1136/adc.2005.082248

S cientific ethics committees (EC) are the foundation of the institutional review process, which is considered an essential barrier before clinical and laboratory research can occur. In layman's terms, EC are to research what the customs department is to immigration. They provide the fundamental checks and balances that provide members of any community with the reassurance that no harm will come to them.

Over the past 10 years, tornado like winds of change have blown through the scientific community, adding strata of documentation and justification for conducting research. Such recent innovations were believed essential to avoid rogue researchers terrorising the innocent. Institutional review is now considered a multi-layered process for our multicultural society. Representation on EC has inevitably expanded to include representatives of every possible group potentially disadvantaged by scientists conducting research. It has been suggested that a week's training in critical thinking may be the most essential preparation for membership of an EC. Given the number of courses inflicted on hospital staff (for example, origami as a tool in conflict resolution), this suggestion may give all researchers some cause for optimism.

For young researchers applying for grants, we sound a note of caution. It is not the heavy competition for grants that should concern you. The biggest hurdle, in fact, is the EC that you will face in justifying the rationale and appropriateness of your study. Commonly this involves EC in teaching hospitals. As veterans of rejection, we propose a tailored approach to successfully meet the needs of your EC and allow you to obtain approval for your study, prior to retirement.

As with any hospital strategy, the EC submission should begin scientifically and end pragmatically. Inspired by the Cochrane confabulation, in which derived ratios from readily available data form the basis for the mandatory closing statement, "More studies are needed", we propose four standardised indices to be calculated in any submission for your local EC. The measurement of these indices will allow objective documentation of which EC are performing appropriately as reflected by higher scores for each index.

\section{Ethics ratio (ER)}

The ethics ratio (ER) is defined as the number of pages of the ethics submission divided by the number of pages contained within the successful grant application. The megabyte equivalents may be substituted. As a rule of thumb, if the ratio exceeds 5 , then the application is likely to be approved with minimal delay (that is, months). A ratio less than 3 is unlikely to be successful, on the grounds of being lightweight. This would be the objective benchmark by which EC is compared nationally and internationally. In time, the ER could be considered as analogous to an impact factor for a scientific journal.

$$
\mathrm{ER}=\frac{\text { No. pages in ethics submission }}{\text { No. pages in successful grant }}
$$

\section{Ethics committee correction index (ECCI)}

This refines the ER in relation to the composition of the EC. It takes into consideration both quantity and experience (longevity) of the EC. The ECCI introduces a correction for the potential bias of gender imbalance in local EC which could be reflected in a higher ER. It has been suggested that a female dominated EC has the potential to erroneously increase discussion time and numbers of suggestions for improvement without altering performance. However, this would be offset by a tendency to meet more frequently. Thus, a male dominated EC could have a higher ECCI on the basis of composition and, given difficulties in males adapting to change, would, in all likelihood, have a higher mean age. In contrast, the lower the dollars value of the grant, the higher the ECCI.

$$
\begin{aligned}
& \text { ECCI }=\mathrm{ER} \times \\
& \frac{\% \text { males on Ethics Committee } \times \text { Mean age of Committee }}{\$ \text { Value of the grant }}
\end{aligned}
$$

\section{The researcher frustration index (RFI)}

This has been developed to obviate the need to sweat blood onto the submitted research protocol in a vain attempt to elicit sympathy from the EC reviewers. Indeed, there is no such thing as a perfectly worded research study information letter or consent form. The Forms Subcommittee of the EC is charged with grammatical correctness: the responsibility for finding a missed comma, incorrectly placed semi-colon, or a split infinitive. To overlook such heinous transgressions of the English language would be unthinkable. Consequently, a group of underground scientific anarchists have devised the young researcher's equivalent of The Da Vinci Code: the researcher frustration index (RFI). The RFI equates the number of corrections required with the number of characters contained within the submission, factoring in the length of time taken to inform the researcher of their errant ways. It is expressed as:

$$
\begin{aligned}
& \text { RFI }=\frac{E R}{4.38} \times \\
& \frac{\text { No. corrections } \times \text { Days to notify corrections }}{\text { No. characters in original grant }}
\end{aligned}
$$

\section{Odds ratio of obligatory revision $\left(O R^{2}\right)$}

It has been decreed by a higher authority that there is no such thing as a perfect submission to an EC. Consequently, revisions will be mandatory but, with the right strategy, resubmission may be avoided. This may be achieved by incorporating a minimum weight of $758 \mathrm{~g}$ per copy of the submission, using a reading age of less than 12 years for the lay community summary, emphasising the potential benefits to the hospital in dollar terms and publicity for the administration on the cover sheet, incorporating as many references as possible to clinical and research governance in 
methodology, and a written assurance of funding which will guarantee the hospital a $20 \%$ facility fee for conducting the study under its auspices. Such measures may reduce the odds ratio for the likelihood of obligatory resubmission $\left(\mathrm{OR}^{2}\right)$.

\section{Preliminary feedback on the ratios from our EC}

In a written preliminary response from our EC, four months after our request for comment on these innovative ratios, we were advised that without sizable external funding the hospital was unwilling to consider the impact of an internationally calibrated standard on its mission. The matter had been referred to the "Clinical Governance Committee" of the hospital, together with a recommendation for a review of our clinical privileges.

\section{Authors' affiliations}

D A Fitzgerald, Department of Respiratory Medicine, Children's Hospital at Westmead, Sydney, Australia

D Isaacs, Department of Infectious Diseases, Children's Hospital at Westmead, Sydney, Australia

A Kemp, Department of Allergy, Immunology and Infectious Disease, Children's Hospital at Westmead, Sydney, Australia

Competing interests: none declared

Correspondence to: Associate Professor D A Fitzgerald, Paediatric Respiratory and Sleep Physician, Dept of Respiratory Medicine, The Children's Hospital at Westmead, Sydney, Locked Bag 4001,

Westmead, Sydney, NSW, Australia 2145; dominif2@chw.edu.au

Accepted 29 June 2005

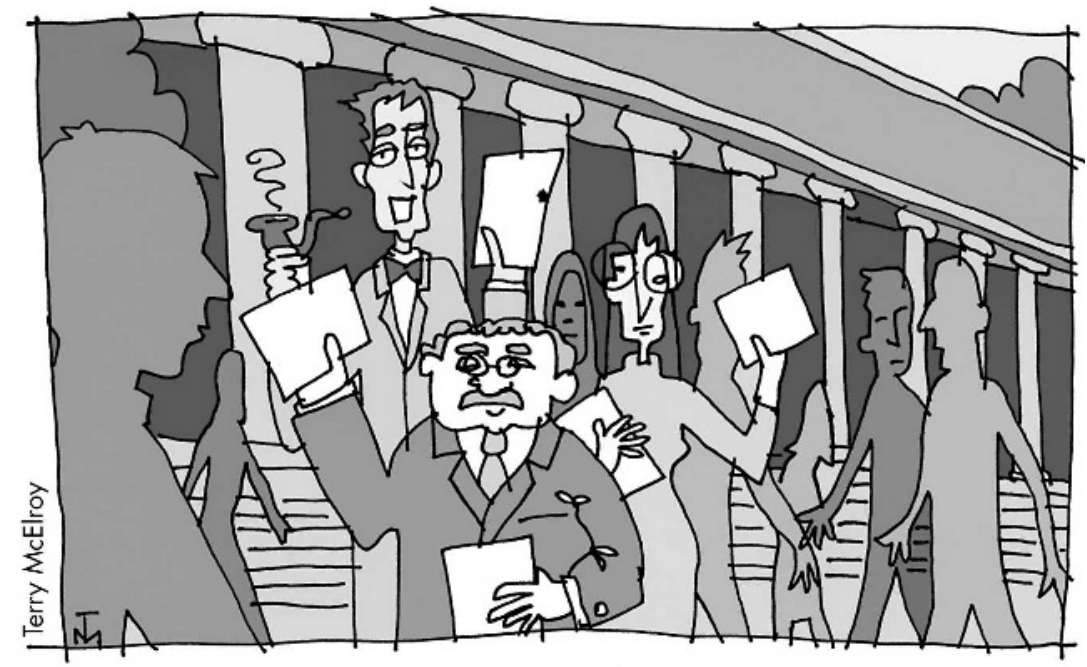

